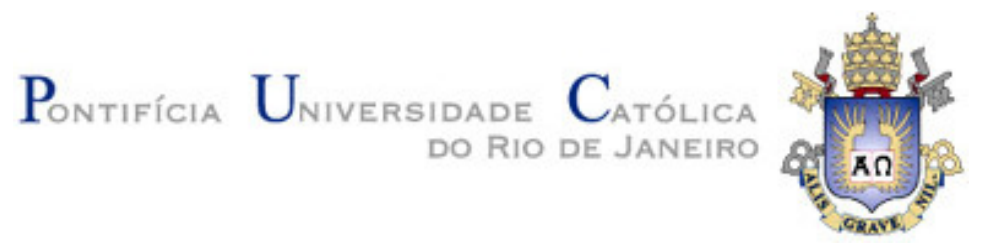

Jelitza Luz Ceballos Infantes

\begin{abstract}
Determinação de Áreas de Controle de Tensão com Base na Interdependência dos Equipamentos Controladores
\end{abstract}

Dissertação de Mestrado

Dissertação apresentada como requisito parcial para obtenção do título de Mestre pelo Programa de PósGraduação em Engenharia Elétrica da PUC-Rio.

Orientador: Prof. Ricardo Bernardo Prada

Co-Orientador: Prof. João Alberto Passos Filho

Rio de Janeiro

Março de 2011 


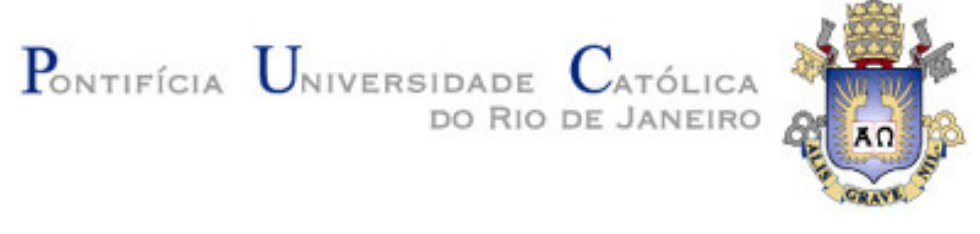

Jelitza Luz Ceballos Infantes

\title{
Determinação de Áreas de Controle de Tensão com Base na Interdependência dos Equipamentos Controladores
}

Dissertação apresentada como requisito parcial para obtenção do grau de Mestre pelo Programa de PósGraduação em Engenharia Elétrica do Departamento de Engenharia Elétrica do Centro Técnico Científico da PUC-Rio. Aprovada pela Comissão Examinadora abaixo assinada.

\author{
Prof. Ricardo Bernardo Prada \\ Orientador \\ Departamento de Engenharia Elétrica - PUC-Rio \\ Prof. João Alberto Passos Filho \\ Co-Orientador \\ UFJF
}

Prof. Ricardo Mota Henriques

CEPEL

Prof. Luiz Cláudio de Araujo Pereira

ONS

Prof. José Eugenio Leal

Coordenador Setorial do Centro

Técnico Científico - PUC-Rio

Rio de Janeiro, 17 de março de 2011 
Todos os direitos reservados. É proibida a reprodução total ou parcial do trabalho sem autorização da universidade, da autora e do orientador.

\section{Jelitza Luz Ceballos Infantes}

Graduada em Engenharia Elétrica na Universidade Nacional de San Agustín (UNSA - Arequipa, Peru) em 2009. Dedicada a tempo integral à pesquisa em Sistemas de Energia Elétrica na PUC-Rio, Brasil.

Ficha Catalográfica

Ceballos Infantes, Jelitza Luz

Determinação de áreas de controle de tensão com base na interdependência dos equipamentos controladores / Jelitza Luz ; orientadores: Ricardo Bernardo Prada , João Alberto Passos Filho. 2011.

207 f. : il. (color.) ; $30 \mathrm{~cm}$

Dissertação (mestrado) - Pontifícia Universidade Católica do Rio de Janeiro, Departamento de Engenharia Elétrica, 2011.

Inclui bibliografia

1. Engenharia elétrica - Teses. 2. Controle de tensão. 3. Áreas de controle de tensão. 4. Equipamentos controladores de tensão. 5. Autovalores e autovetores. I. Prada, Ricardo Bernardo. II. Passos Filho, João Alberto. III. Pontifícia Universidade Católica do Rio de Janeiro. Departamento de Engenharia Elétrica. IV. Título.

CDD: 621.3 
Dedico este trabalho à memória de meus amados avôs: Juan e Rosário. 


\section{Agradecimentos}

A Deus, por sua presença e direção em cada instante de minha vida, por todas as bondades e privilégios com que abençoa minha existência.

A minha amada família: meus pais Amparo e Leoncio, minha irmã Militza, meus tios Mario e Vladimir, pelo amor, bons conselhos, apoio constante e por acreditar em mim, sempre.

Meu profundo agradecimento aos meus queridos orientadores: Ricardo Bernardo Prada e João Alberto Passos Filho, pela confiança, permanente apoio, orientação e paciência durante as diferentes etapas do desenvolvimento desta Dissertação.

Aos respeitados membros da Comissão Julgadora: Ricardo Mota Henriques e Luiz Cláudio de Araujo, pelas inestimáveis colaborações nesta Dissertação.

À CNPq e à PUC-Rio, pela oportunidade de estudar meu Mestrado no Brasil e pelos auxílios financeiros concedidos.

Ao CEPEL pela licença de uso do programa computacional ANAREDE.

Meu reconhecimento e gratidão aos professores do DEE, especialmente ao Professor Eduardo Pires de Souza, pelos ensinamentos e ajuda.

A todos meus amigos do curso de Pós-Graduação, muito especialmente ao Carlos Portugal, Javier Ortega, Josué Uscata, Omar Gálvez, Maritza Arapa, Edwin Valderrama Silvino da Costa, Fabio Bicalho, e outros tantos que, de alguma forma, estiveram presentes com sua colaboração e amizade. 


\section{Resumo}

Ceballos Infantes, Jelitza Luz; Prada, Ricardo Bernardo; Passos Filho, João Alberto. Determinação de Áreas de Controle de Tensão com Base na Interdependência dos Equipamentos Controladores. Rio de Janeiro, 2011. 207p. Dissertação de Mestrado - Departamento de Engenharia Elétrica, Pontifícia Universidade Católica do Rio de Janeiro.

Após a incidência de inúmeros problemas relacionados a fenômenos de instabilidade de tensão, o controle de potência reativa em sistemas elétricos de potência tornou-se um assunto importante os últimos anos. Um adequado controle do perfil de tensão em uma área pode contribuir para evitar este tipo de problema. Com esse objetivo, determinam-se áreas de controle de tensão a partir da análise dos autovalores e autovetores das matrizes de sensibilidade: VCS "Voltage Control Sensitivity Matrix" e QV. A matriz de sensibilidade [VCS] é constituída por elementos diagonais que relacionam a grandeza controladora de cada equipamento com a respectiva tensão controlada (variável controlada), e a análise do sinal desses elementos estabelece se uma determinada ação de controle será adequada ou não, isto é, se terá efeito esperado ou oposto. Os elementos fora da diagonal representam a interdependência existente entre os equipamentos controladores de tensão. A matriz de sensibilidade QV, nomeada como [ $\mathrm{J}_{\mathrm{Sov}}$ ] é obtida a partir da matriz Jacobiana do sistema linearizado das equações de fluxo de carga. As áreas de controle de tensão determinadas da análise por autovalores e autovetores usando-se cada uma das matrizes de sensibilidade são coerentes. Adicionalmente, obtêm-se áreas de controle de tensão diretamente das matrizes de sensibilidade. Estas áreas foram comparadas encontrando-se resultados coerentes.

\section{Palavras - chave}

Controle de tensão; áreas de controle de tensão; equipamentos controladores de tensão; autovalores e autovetores. 


\section{Abstract}

Ceballos Infantes, Jelitza Luz; Prada, Ricardo Bernardo (Advisor); Passos Filho, João Alberto. Determination of Voltage Control Areas based on Interdependent Controller Equipments. Rio de Janeiro, 2011. 207p. MSc Dissertation Departamento de Engenharia Elétrica, Pontifícia Universidade Católica do Rio de Janeiro.

After of the incidence of innumerable problems related to voltage instability phenomena, the control of reactive power in electrical power systems became an important issue in the last years. The adequate control of the voltage for a specific area can prevent this kind of problem. With this objective, voltage control areas are established from an eigenvalues and eigenvectors analysis of the sensitivity matrixes: VCS "Voltage Control Sensitivity Matrix" and QV. The sensitive matrix [VCS] is form by diagonal elements that relate to the controlling variables and to the controlled voltage (controlled variable), and the analysis of the sign of each diagonal element indicate if a specific control action is adequate or not. The off-diagonal elements represent the interdependence among the voltage controller equipments. The sensitivity matrix QV, called $\left[\mathrm{J}_{\mathrm{SQv}}\right]$ is obtained from the Jacobean matrix from the linear load flow equations. The voltage control areas recognized from the eigenvalues and eigenvectors analysis to each sensitivity matrix are coherent. Also, voltage control areas were identified directly from sensitivity matrixes. These areas were compared founded coherent results.

\section{Keywords}

Voltage control; voltage control areas; voltage controller equipments; eigenvalues and eigenvectors. 


\section{Sumário}

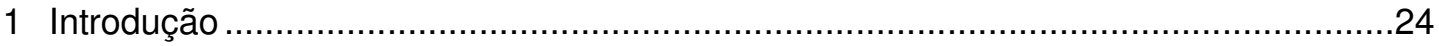

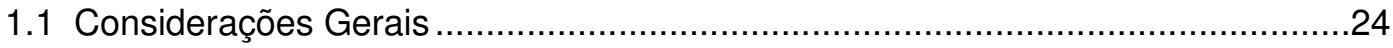

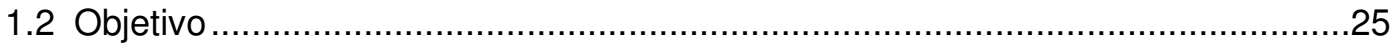

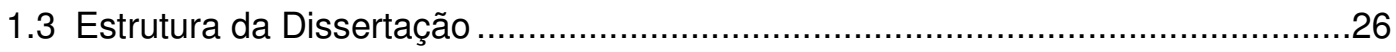

2 Matriz de Sensibilidade dos Controles de Tensão ....................................................28

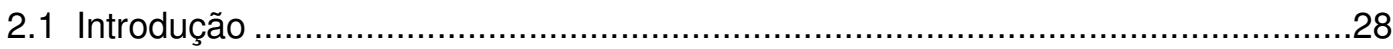

2.2 Matriz de Sensibilidade dos Controles de Tensão Incluindo Modelo

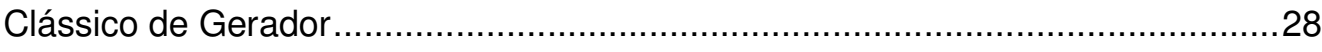

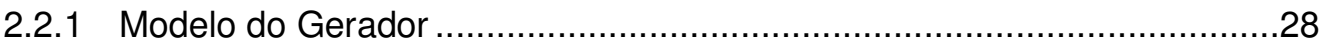

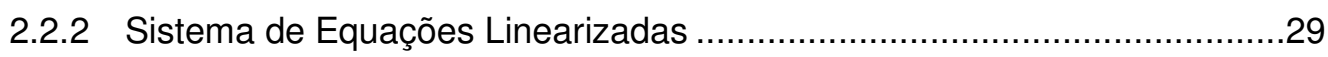

2.2.3 Aplicação em Sistemas-Teste .................................................................

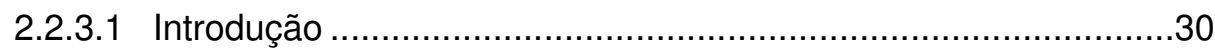

2.2.3.2 Sistema-Teste de 5 Barras ........................................................30

2.3 Matriz de Sensibilidade dos Controles de Tensão Incluindo Novo

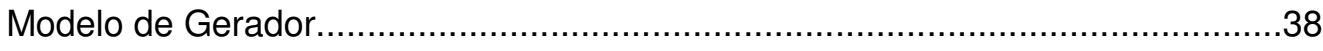

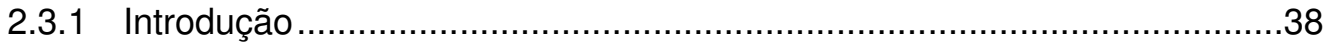

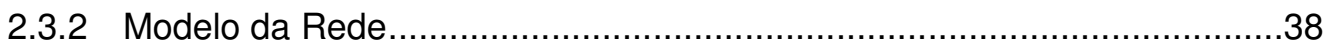

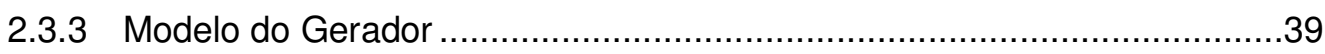

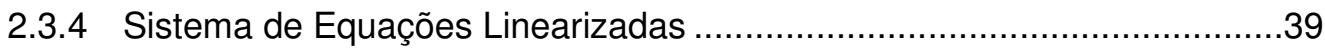

2.3.5 Aplicação em Sistemas-Teste ..............................................................42

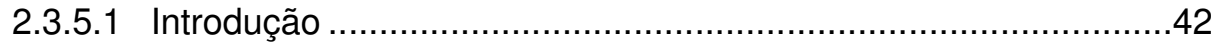

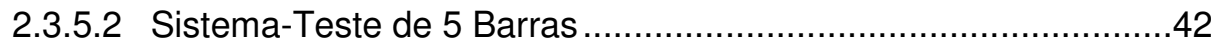

2.4 Comparação entre as Matrizes de Sensibilidade dos Controles de Tensão obtidas com os Dois Modelos de Gerador .............................................48

2.5 Determinação de Áreas de Controle de Tensão com Base na Matriz de Sensibilidade dos Controles de Tensão..........................................................

2.5.1 Análise Modal de uma Matriz .............................................................. 49

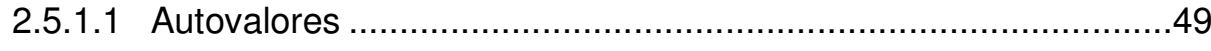

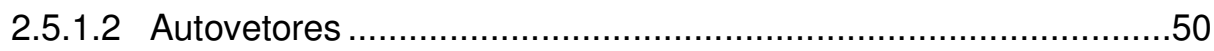

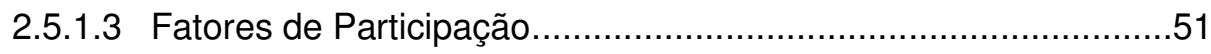

2.6 Resultados da Definição de Áreas de Controle de Tensão...................................54

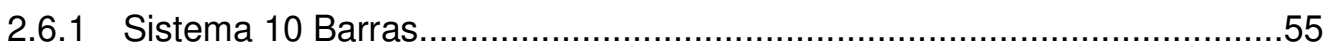


2.6.1.1 Baixa Impedância de Interligação.........................................56

2.6.1.2 Alta Impedância de Interligação.............................................58

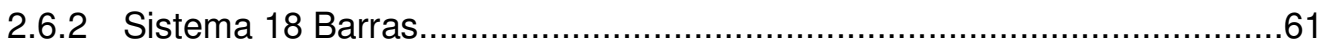

2.6.2.1 Baixa Impedância de Interligação.........................................62

2.6.2.2 Alta Impedância de Interligação............................................66

2.6.3 Sistema New England 39 Barras....................................................71

2.6.4 Sistema IEEE 118 Barras..................................................................... 75

2.6.5 Sistema S/SE Brasileiro 730 Barras ...............................................78

2.7 Identificação de Áreas de Controle Diretamente da Matriz de Sensibilidade dos Controles de Tensão............................................................ 82

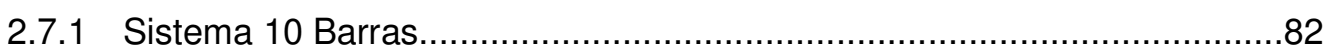

2.7.1.1 Baixa Impedância de Interligação.........................................82

2.7.1.2 Alta Impedância de Interligação...........................................83

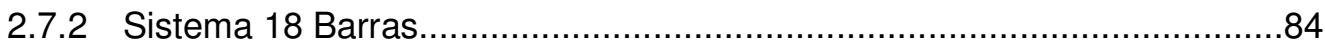

2.7.2.1 Baixa Impedância de Interligação...........................................84

2.7.2.2 Alta Impedância de Interligação.............................................85

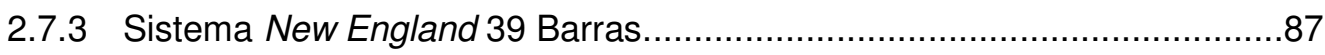

2.7.4 Sistema IEEE 118 Barras............................................................ 88

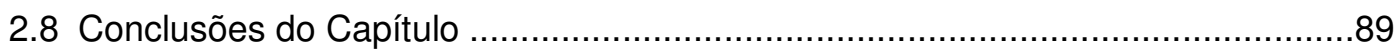

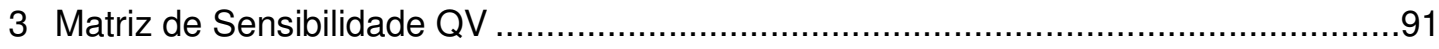

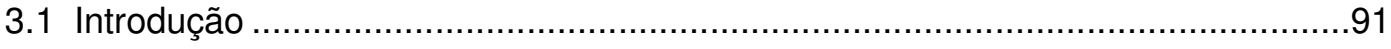

3.2 Cálculo da Matriz de Sensibilidade [JSQV] ................................................

3.3 Determinação de Áreas de Controle de Tensão com Base na Matriz de

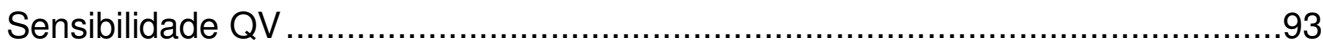

3.3.1 Método de Determinação de Áreas de Controle de Tensão ......................93

3.3.2 Algoritmo de Busca de Áreas de Controle de Tensão ................................94

3.4 Resultados da Definição de Áreas de Controle de Tensão .....................................95

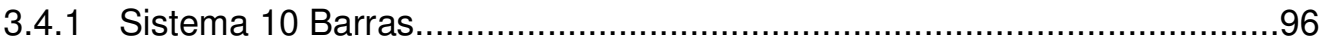

3.4.1.1 Baixa Impedância de Interligação.............................................97

3.4.1.2 Alta Impedância de Interligação.........................................101

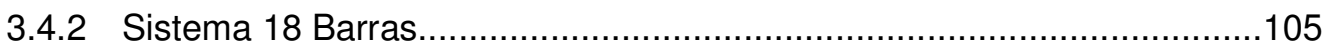

3.4.2.1 Baixa Impedância de Interligação..........................................106

3.4.2.2 Alta Impedância de Interligação...........................................110

3.4.3 Sistema New England 39 Barras.....................................................114

3.4.4 Sistema IEEE 118 Barras............................................................119 
3.4.5 Sistema S/SE Brasileiro 730 Barras

3.5 Identificação de Áreas de Controle de Tensão Diretamente da Matriz

de Sensibilidade QV 128

3.5.1 Sistema 10 Barras.................................................................. 128

3.5.1.1 Baixa Impedância de Interligação.......................................128

3.5.1.2 Alta Impedância de Interligação............................................129

3.5.2 Sistema 18 Barras.......................................................................... 130

3.5.2.1 Baixa Impedância de Interligação........................................130

3.5.2.2 Alta Impedância de Interligação..........................................130

3.5.3 Sistema New England 39 Barras....................................................131

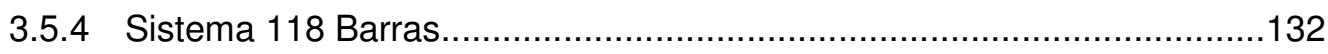

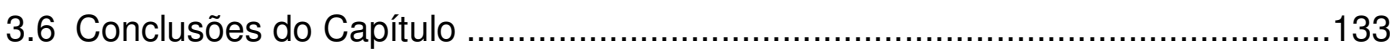

4 Comparação entre os Métodos de Determinação de Áreas de Controle de

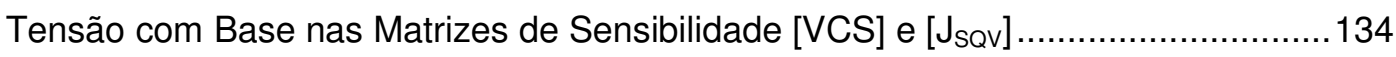

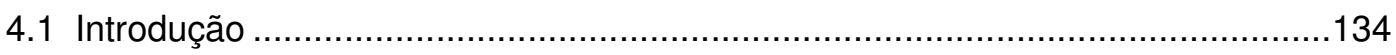

4.2 Comparação entre os Métodos................................................................134

4.2.1 Primeiro Critério de Comparação: Autovalores Críticos .........................134

4.2.1.1 Autovalores Críticos na Matriz [VCS] ...................................134

4.2.1.2 Autovalores Críticos na Matriz [JSQv] ...................................138

4.2.2 Segundo Critério de Comparação: Tipo de Barras que Compõem as Áreas de Controle de Tensão .......................................................142

4.2.2.1 Tipos de Barras na Matriz [VCS] .......................................142

4.2.2.2 Tipos de Barras na Matriz $\left[\mathrm{J}_{\mathrm{SQv}}\right]$.........................................143

4.2.3 Terceiro Critério de Comparação: Sensibilidade entre as Barras .............145

4.2.4 Quarto Critério de Comparação: Quantidade de Barras Analisadas........145

4.2.5 Quinto Critério de Comparação: Algoritmo de Busca de Áreas de

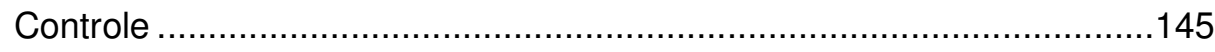

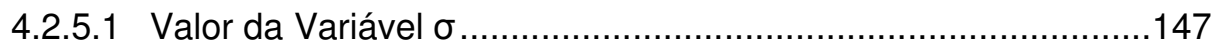

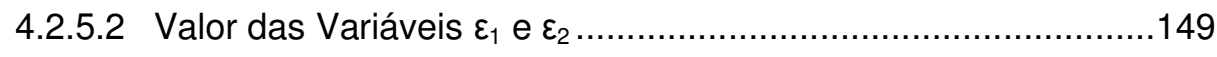

4.2.5.3 Subáreas de Controle de Tensão .........................................151

4.2.5.4 Barras que Não Pertencem a Nenhuma Área de Controle ........155

4.2.5.5 Incoerência de Barras que Aparecem Incluídas em uma Área de Controle ................................................................ 158

4.2.5.6 Barras que se Repetem em Duas Áreas de Controle ................161

4.2.6 Sexto Critério de Comparação: Áreas de Controle de Tensão ..................163 
4.2.6.1 Áreas de Controle de Tensão no Sistema 10 Barras .................164

4.2.6.2 Áreas de Controle de Tensão no Sistema 18 Barras ..................165

4.2.6.3 Áreas de Controle de Tensão no Sistema New England

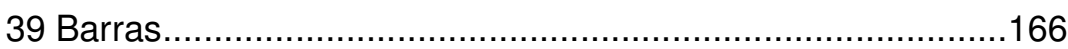

4.2.6.4 Áreas de Controle de Tensão no Sistema IEEE 118 Barras. 168

4.2.6.5 Áreas de Controle de Tensão no Sistema S/SE Brasileiro 730 Barras 168

4.3 Conclusões do Capítulo 170

5 Conclusões e Propostas para Trabalhos Futuros. 172

5.1 Conclusões 172

5.2 Trabalhos Futuros 174

Referências Bibliográficas 175

Apêndice A - Revisão do Método de Newton-Raphson para Solução do Fluxo de Potência 177

Apêndice B - Arquivos de Dados de Entrada para o ANAREDE (Matriz [VCS]) ............182

B.1 Sistema-Teste 10 Barras ......................................................182

B.2 Sistema-Teste 18 Barras .......................................................184

B.3 Sistema-Teste New England 39 Barras ...................................186

B.4 Sistema-Teste IEEE 118 Barras ..............................................189

Apêndice C - Arquivos de Dados de Entrada para o ANAREDE (Matriz [J $\left.\mathrm{JQv}_{\text {SQ }}\right]$ )............198

C.1 Sistema-Teste 10 Barras ....................................................198

C.2 Sistema-Teste 18 Barras .....................................................198

C.3 Sistema-Teste New England 39 Barras ...................................199

C.4 Sistema-Teste IEEE 118 Barras .............................................201 


\section{Lista de Tabelas}

Tabela 2.1 - Dados do Sistema-Teste de 5 Barras ...................................................... 31

Tabela 2.2 - Valores de Eg Calculados com (2.5), (2.6) e (2.7) ........................................32

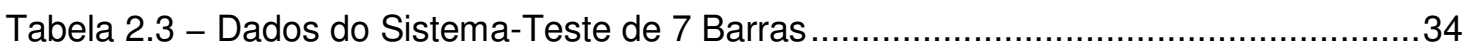

Tabela 2.4 - Dados do Sistema-Teste de 7 Barras / Região B ........................................35

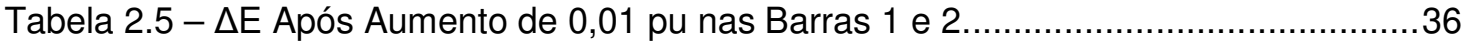

Tabela 2.6 - $\Delta$ E Após Redução de 0,01 pu nas Barras 1 e 2 ...........................................36

Tabela 2.7 - $\Delta$ E Após Aumento de 0,01 pu nas Barras 1 e 2 / Região B ..........................37

Tabela $2.8-\Delta$ E Após Redução de 0,01 pu nas Barras 1 e 2 / Região B ..........................38

Tabela 2.9 - Dados do Sistema-Teste de 5 Barras ......................................................44

Tabela 2.10 - Dados do Sistema-Teste de 5 Barras / Região B .....................................45

Tabela 2.11 - $\Delta$ Eq Após Aumento de 0,01 pu na Tensão de Referência das Barras

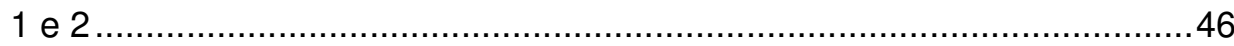

Tabela $2.12-\Delta$ Eq Após Redução de 0,01 pu na Tensão de Referência das Barras

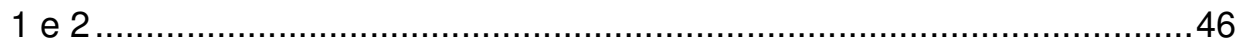

Tabela $2.13-\Delta$ Eq Após Aumento de 0,01 pu na Tensão de Referência das Barras 1 e 2 / Região B................................................................................4 4

Tabela 2.14 - $\Delta$ Eq Após Redução de 0,01 pu na Tensão de Referência das Barras 1 e 2 / Região B............................................................................. 48

Tabela 2.15 - Autovalores da Matriz [VCS] do Sistema 10 Barras (jX=0,01\%) ................56

Tabela 2.16 - Autovalores da Matriz [VCS] do Sistema 10 Barras (jX=10000\%) ..............59

Tabela 2.17 - Autovalores da Matriz [VCS] do Sistema 18 Barras (jX=0,01\%) ................63

Tabela 2.18 - Autovalores da Matriz [VCS] do Sistema 18 Barras (jX=10000\%) ..............67

Tabela 2.19 - Características Principais do Sistema New England 39 Barras ..................71

Tabela 2.20 - Autovalores da Matriz [VCS] do Sistema 39 Barras..................................72

Tabela 2.21 - Características Principais do Sistema IEEE 118 Barras .............................75

Tabela 2.22 - Autovalores da Matriz [VCS] do Sistema IEEE 118 Barras.........................76

Tabela 2.23 - Características Principais do Sistema S/SE Brasileiro 730 Barras...............79

Tabela 2.24 - Autovalores da Matriz [VCS] do Sistema S/SE Brasileiro 730 Barras ..........79

Tabela 2.25 - Áreas de Controle do Sistema S/SE Brasileiro 730 Barras .........................79

Tabela 2.26 - Comparação entre as Áreas de Controle de Tensão obtidas para o

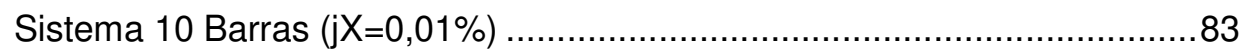

Tabela 2.27 - Comparação entre as Áreas de Controle de Tensão obtidas para o Sistema 10 Barras $(\mathrm{j}=10000 \%)$ .84 
Tabela 2.28 - Comparação entre as Áreas de Controle de Tensão obtidas para o

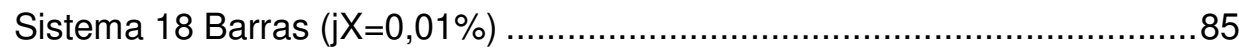

Tabela 2.29 - Comparação entre as Áreas de Controle de Tensão obtidas para o Sistema 18 Barras ( $\mathrm{X}=10000 \%)$. 87

Tabela 2.30 - Comparação entre as Áreas de Controle de Tensão obtidas para o Sistema 39 Barras 88

Tabela 2.31 - Comparação entre as Áreas de Controle de Tensão para o Sistema 118 Barras 89

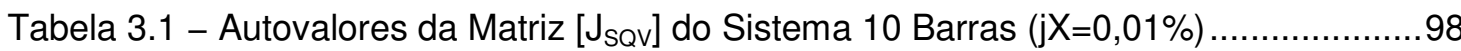

Tabela 3.2 - Autovalores da Matriz [ $\left.\mathrm{J}_{\mathrm{SQv}}\right]$ do Sistema 10 Barras $(\mathrm{jX}=10000 \%) \ldots \ldots \ldots \ldots . . . . .102$

Tabela 3.3 - Autovalores da Matriz [ $\left.\mathrm{J}_{\mathrm{SQv}}\right]$ do Sistema 18 Barras $(\mathrm{jX}=0,01 \%) \ldots \ldots \ldots \ldots \ldots . . . . .106$

Tabela 3.4 - Autovalores da Matriz [Jsov] do Sistema 18 Barras (jX=10000\%) ...............111

Tabela 3.5 - Características Principais do Sistema New England 39 Barras ..................115

Tabela 3.6 - Autovalores da Matriz [JSQv] do Sistema 39 Barras ..................................116

Tabela 3.7 - Características Principais do Sistema IEEE 118 Barras .............................120

Tabela 3.8 - Autovalores da Matriz [J SQv $_{\text {] }}$ do Sistema IEEE 118 Barras .......................121

Tabela 3.9 - Características Principais do Sistema S/SE Brasileiro 730 Barras...............124

Tabela 3.10 - Autovalores da Matriz [J Jsv] do Sistema S/SE Brasileiro 730 Barras .........124

Tabela 3.11 - Áreas de Controle do Sistema S/SE Brasileiro 730 Barras ........................124

Tabela 3.12 - Comparação entre as Áreas de Controle de Tensão obtidas para o Sistema 10 Barras $(\mathrm{j} X=0,01 \%)$ 129

Tabela 3.13 - Comparação entre as Áreas de Controle de Tensão obtidas para o Sistema 10 Barras ( $\mathrm{X}=10000 \%)$

Tabela 3.14 - Comparação entre as Áreas de Controle de Tensão obtidas para o Sistema 18 Barras ( $\mathrm{X}=0,01 \%)$ 130

Tabela 3.15 - Comparação entre as Áreas de Controle de Tensão obtidas para o Sistema 18 Barras ( $\mathrm{X}=10000 \%)$ 131

Tabela 3.16 - Comparação entre as Áreas de Controle de Tensão obtidas para o Sistema 39 Barras 131

Tabela 3.17 - Comparação entre as Áreas de Controle de Tensão para o Sistema 118 Barras. 132

Tabela 4.1 - Áreas de Controle a partir da Matriz [VCS] para o Sistema de 39 Barras 142

Tabela 4.2 - Áreas de Controle a partir da Matriz [J $\mathrm{J}_{\mathrm{sqv}}$ ] para o Sistema de 39 Barras.....144 


\section{Lista de Figuras}

Figura 2.1 - Diagrama Unifilar do Modelo de Regime Permanente do Gerador .................28

Figura 2.2 - Nomenclatura das Barras de Geração ..........................................................29

Figura 2.3 - Diagrama Unifilar do Sistema-Teste de 5 Barras.......................................31

Figura 2.4 - Circuito Elétrico do Modelo Clássico de Gerador ........................................32

Figura 2.5 - Diagrama Unifilar do Sistema-Teste de 5 Barras incluindo o Modelo

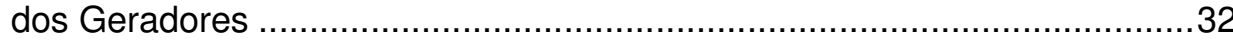

Figura 2.6 - Diagrama Unifilar do Sistema-Teste de 5 Barras.......................................42

Figura 2.7 - Fluxograma Simplificado da Busca de Áreas de Controle pelos Maiores .......54

Figura 2.8 - Diagrama Unifilar do Sistema 10 Barras incluindo Modelo Clássico de Gerador .55

Figura 2.9 - Mode-Shape de $\lambda_{1}$ da Matriz [VCS] para o Sistema 10 Barras $(\mathrm{jX}=0,01 \%)$

Figura 2.10 - Mode-Shape de $\lambda_{2}$ da Matriz [VCS] para o Sistema 10 Barras

$$
(\mathrm{jX}=0,01 \%)
$$

Figura 2.11 - Áreas de Controle de Tensão a partir da Matriz [VCS] para o Sistema 10 Barras que inclui Modelo Clássico de Gerador com jX=0,01\%

Figura 2.12 - Mode-Shape de $\lambda_{1}$ da Matriz [VCS] para o Sistema 10 Barras (jX=10000\%)

Figura 2.13 - Mode-Shape de $\lambda_{2}$ da Matriz [VCS] para o Sistema 10 Barras (jX=10000\%)

Figura 2.14 - Áreas de Controle de Tensão a partir da Matriz [VCS] para o Sistema 10 Barras com jX=10000\%, incluindo Modelo Clássico de Gerador .... 61

Figura 2.15 - Diagrama Unifilar do Sistema 18 Barras incluindo Modelo Clássico de Gerador 62

Figura 2.16 - Mode-Shape de $\lambda_{1}$ da Matriz [VCS] para o Sistema 18 Barras $(\mathrm{j} X=0,01 \%)$ 64

Figura 2.17 - Mode-Shape de $\lambda_{2}$ da Matriz [VCS] para o Sistema 18 Barras

$$
\text { (jX=0,01\%) . }
$$

Figura 2.18 - Mode-Shape de $\lambda_{3}$ da Matriz [VCS] para o Sistema 18 Barras

$$
(\mathrm{XX}=0,01 \%)
$$

Figura 2.19 - Mode-Shape de $\lambda_{4}$ da Matriz [VCS] para o Sistema 18 Barras

$$
(\mathrm{jX}=0,01 \%)
$$


Figura 2.20 - Áreas de Controle de Tensão a partir da Matriz [VCS] para o Sistema 18 Barras que inclui Modelo Clássico de Gerador com jX=0,01\% 66

Figura 2.21 - Mode-Shape de $\lambda_{1}$ da Matriz [VCS] para o Sistema 18 Barras (jX=10000\%) 68

Figura 2.22 - Mode-Shape de $\lambda_{2}$ da Matriz [VCS] para o Sistema 18 Barras (jX=10000\%)

Figura 2.23 - Mode-Shape de $\lambda_{3}$ da Matriz [VCS] para o Sistema 18 Barras

$$
\text { (jX=10000\%) }
$$

Figura 2.24 - Mode-Shape de $\lambda_{4}$ da Matriz [VCS] para o Sistema 18 Barras

$$
\text { (jX=10000\%) }
$$

Figura 2.25 - Áreas de Controle de Tensão a partir da Matriz [VCS] para o Sistema 18 Barras que inclui Modelo Clássico de Gerador com jX=10000\% ............70

Figura 2.26 - Diagrama Unifilar do Sistema New England 39 Barras 71

Figura 2.27 - Mode-Shape de $\lambda_{1}$ da Matriz [VCS] para o Sistema New England 39 Barras 72

Figura 2.28 - Mode-Shape de $\lambda_{2}$ da Matriz [VCS] para o Sistema New England 39 Barras .....

Figura 2.29 - Mode-Shape de $\lambda_{3}$ da Matriz [VCS] para o Sistema New England 39 Barras .73

Figura 2.30 - Áreas de Controle de Tensão a partir da Matriz [VCS] para o Sistema New England 39 Barras...... 74

Figura 2.31 - Diagrama Unifilar do Sistema IEEE 118 Barras.......................................75

Figura 2.32 - Mode-Shape de $\lambda_{1}$ da Matriz [VCS] para o Sistema IEEE 118 Barras ..........76

Figura 2.33 - Mode-Shape de $\lambda_{2}$ da Matriz [VCS] para o Sistema IEEE 118 Barras .........77

Figura 2.34 - Mode-Shape de $\lambda_{3}$ da Matriz [VCS] para o Sistema IEEE 118 Barras ..........77

Figura 2.35 - Áreas de Controle de Tensão a partir da Matriz [VCS] para o Sistema IEEE 118 Barras. 78

Figura 2.36 - Áreas de Controle de Tensão a partir da Matriz [VCS] para o Sistema S/SE Brasileiro 730 Barras. 81

Figura 3.1 - Fluxograma Simplificado da Busca de Áreas de Controle pelos

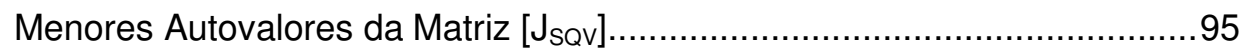

Figura 3.2 - Diagrama Unifilar do Sistema 10 Barras...............................................96

Figura 3.3 - Mode-Shape de $\lambda_{1}$ da Matriz [ $\mathrm{J}_{\mathrm{SQv}}$ ] para o Sistema 10 Barras

$$
(\mathrm{jX}=0,01 \%) \text {.... }
$$

Figura 3.4 - Mode-Shape de $\lambda_{2}$ da Matriz [ $\mathrm{J}_{\mathrm{SQv}}$ ] para o Sistema 10 Barras

$$
(\mathrm{JX}=0,01 \%)
$$


Figura 3.5 - Áreas de Controle de Tensão a partir da Matriz [ $\left.\mathrm{J}_{\mathrm{SQv}}\right]$ para o Sistema 10 Barras com jX=0,01\%................................................................ 100

Figura 3.6 - Mode-Shape de $\lambda_{3}$ da Matriz [ $\mathrm{J}_{\mathrm{SQv}}$ ] para o Sistema 10 Barras

$$
(\mathrm{jX}=0,01 \%)
$$

Figura 3.7 - Mode-Shape de $\lambda_{4}$ da Matriz [ $\mathrm{J}_{\mathrm{SQv}}$ ] para o Sistema 10 Barras

$$
(\mathrm{jX}=0,01 \%)
$$

Figura 3.8 - Mode-Shape de $\lambda_{1}$ da Matriz [ $\mathrm{J}_{\mathrm{SQv}}$ ] para o Sistema 10 Barras

$$
\text { (jX=10000\%) }
$$

Figura 3.9 - Mode-Shape de $\lambda_{2}$ da Matriz [J $\mathrm{J}_{\mathrm{SQv}}$ ] para o Sistema 10 Barras

$$
\text { (jX=10000\%) }
$$

Figura 3.10 - Áreas de Controle de Tensão a partir da Matriz [J $\left.\mathrm{J}_{\mathrm{SQv}}\right]$ para o Sistema 10 Barras com $\mathrm{jX}=10000 \%$. 104

Figura 3.11 - Mode-Shape de $\lambda_{3}$ da Matriz [J $\mathrm{J}_{\mathrm{SQv}}$ ] para o Sistema 10 Barras

$$
\text { (jX=10000\%) }
$$

Figura 3.12 - Mode-Shape de $\lambda_{4}$ da Matriz [J $\mathrm{J}_{\mathrm{sov}}$ ] para o Sistema 10 Barras

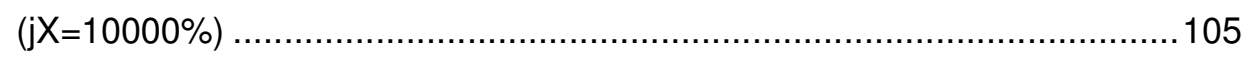

Figura 3.13 - Diagrama Unifilar do Sistema 18 Barras............................................ 106

Figura 3.14 - Mode-Shape de $\lambda_{1}$ da Matriz [J $\mathrm{J}_{\mathrm{Sov}}$ ] para o Sistema 18 Barras

$$
(\mathrm{jX}=0,01 \%)
$$

Figura 3.15 - Mode-Shape de $\lambda_{2}$ da Matriz [J $\mathrm{J}_{\mathrm{Sov}}$ para o Sistema 18 Barras

$$
(\mathrm{j} X=0,01 \%)
$$

Figura 3.16 - Mode-Shape de $\lambda_{3}$ da Matriz [J $\mathrm{J}_{\mathrm{SQv}}$ ] para o Sistema 18 Barras

$$
\text { (jX=0,01\%) }
$$

Figura 3.17 - Mode-Shape de $\lambda_{4}$ da Matriz [ $\mathrm{J}_{\mathrm{SQv}}$ ] para o Sistema 18 Barras

$$
\text { (jX=0,01\%) }
$$

Figura 3.18 - Áreas de Controle de Tensão a partir da Matriz [J $\left.\mathrm{J}_{\mathrm{SQv}}\right]$ para o Sistema 18 Barras com $\mathrm{jX}=0,01 \%$

Figura 3.19 - Mode-Shape de $\lambda_{1}$ da Matriz [J $\mathrm{J}_{\mathrm{SQv}}$ ] para o Sistema 18 Barras

$$
\text { (jX=10000\%) }
$$

Figura 3.20 - Mode-Shape de $\lambda_{2}$ da Matriz [ $\mathrm{J}_{\mathrm{SQv}}$ ] para o Sistema 18 Barras

$$
\text { (jX=10000\%) }
$$

Figura 3.21 - Mode-Shape de $\lambda_{3}$ da Matriz [J $\mathrm{J}_{\mathrm{SQv}}$ ] para o Sistema 18 Barras

$$
\text { (jX=10000\%) }
$$

Figura 3.22 - Mode-Shape de $\lambda_{4}$ da Matriz [J $\mathrm{J}_{\mathrm{sov}}$ ] para o Sistema 18 Barras

$$
(\mathrm{X}=10000 \%)
$$


Figura 3.23 - Áreas de Controle de Tensão a partir da Matriz [ $\left.\mathrm{J}_{\mathrm{SQv}}\right]$ para o Sistema

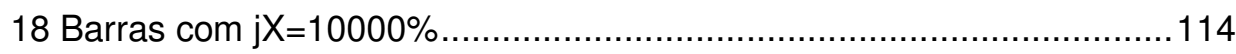

Figura 3.24 - Diagrama Unifilar do Sistema New England 39 Barras.............................115

Figura 3.25 - Mode-Shape de $\lambda_{1}$ da Matriz [ J $\mathrm{J}_{\mathrm{SQv}}$ ] para o Sistema 39 Barras...................116

Figura 3.26 - Mode-Shape de $\lambda_{2}$ da Matriz [J $\mathrm{J}_{\mathrm{SQv}}$ ] para o Sistema 39 Barras...................117

Figura 3.27 - Mode-Shape de $\lambda_{3}$ da Matriz [VCS] para o Sistema 39 Barras ..................118

Figura 3.28 - Áreas de Controle de Tensão a partir da Matriz [J $\left.\mathrm{J}_{\mathrm{SQv}}\right]$ para o Sistema

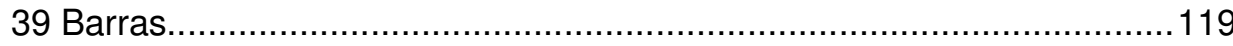

Figura 3.29 - Diagrama Unifilar do Sistema IEEE 118 Barras....................................120

Figura 3.30 - Mode-Shape de $\lambda_{1}$ da Matriz [ $\mathrm{J}_{\mathrm{SQv}}$ ] para o Sistema IEEE 118 Barras.........121

Figura 3.31 - Mode-Shape de $\lambda_{2}$ da Matriz [J Jov] para o Sistema IEEE 118 Barras .........122

Figura 3.32 - Mode-Shape de $\lambda_{3}$ da Matriz [ J $\mathrm{J}_{\mathrm{SQv}}$ ] para o Sistema IEEE 118 Barras.........122

Figura 3.33 - Áreas de Controle de Tensão a partir da Matriz [ $\left.\mathrm{J}_{\mathrm{SQv}}\right]$ para o Sistema IEEE 118 Barras...................................................................................123

Figura 3.34 - Aproximação das Áreas de Controle de Tensão a partir da Matriz [J $\left.\mathrm{J}_{\mathrm{SQv}}\right]$ para o Sistema S/SE Brasileiro 730 Barras....

Figura 4.1 - Diagrama Unifilar do Sistema 10 Barras incluindo Modelo Clássico de Gerador 136

Figura 4.2 - Mode-Shape de $\lambda_{1}$ da Matriz [VCS] para o Sistema 10 Barras (jX=10000\%) 136

Figura 4.3 - Mode-Shape de $\lambda_{2}$ da Matriz [VCS] para o Sistema 10 Barras $(\mathrm{j} X=10000 \%)$ 136

Figura 4.4 - Mode-Shape de $\lambda_{3}$ da Matriz [VCS] para o Sistema 10 Barras

$$
\text { (jX=10000\%) }
$$

Figura 4.5 - Mode-Shape de $\lambda_{4}$ da Matriz [VCS] para o Sistema 10 Barras

$$
\text { (jX=10000\%) }
$$

Figura 4.6 - Áreas de Controle de Tensão a partir da Matriz [VCS] para o Sistema 10 Barras com jX=10000\%, incluindo Modelo Clássico de Gerador ..... 137

Figura 4.7 - Diagrama Unifilar do Sistema 10 Barras. 139

Figura 4.8 - Mode-Shape de $\lambda_{1}$ da Matriz [ $\mathrm{J}_{\mathrm{SQv}}$ ] para o Sistema 10 Barras

$$
\text { (jX=10000\%) }
$$

Figura 4.9 - Mode-Shape de $\lambda_{2}$ da Matriz [ $\mathrm{J}_{\mathrm{SQv}}$ ] para o Sistema 10 Barras

$$
\text { (jX=10000\%) }
$$

Figura 4.10 - Mode-Shape de $\lambda_{3}$ da Matriz [J $\left.\mathrm{J}_{\mathrm{SQv}}\right]$ para o Sistema 10 Barras

$$
(\mathrm{X}=10000 \%)
$$


Figura 4.11 - Mode-Shape de $\lambda_{4}$ da Matriz [ $\mathrm{J}_{\mathrm{SQv}}$ ] para o Sistema 10 Barras

$$
(\mathrm{X}=10000 \%)
$$

Figura 4.12 - Mode-Shape de $\lambda_{5}$ da Matriz [ $\mathrm{J}_{\mathrm{SQv}}$ ] para o Sistema 10 Barras

$$
(\mathrm{X}=10000 \%)
$$

Figura 4.13 - Mode-Shape de $\lambda_{6}$ da Matriz [ $\left.\mathrm{J}_{\mathrm{SQv}}\right]$ para o Sistema 10 Barras

$$
\text { (jX=10000\%) }
$$

Figura 4.14 - Mode-Shape de $\lambda_{7}$ da Matriz [J Jov] para o Sistema 10 Barras

$$
(\mathrm{X}=10000 \%)
$$

Figura 4.15 - Mode-Shape de $\lambda_{8}$ da Matriz [J Jov] para o Sistema 10 Barras

$$
\text { (jX=10000\%) }
$$

Figura 4.16 - Mode-Shape de $\lambda_{9}$ da Matriz [J $\mathrm{J}_{\mathrm{SQv}}$ ] para o Sistema 10 Barras

$$
(\mathrm{X}=10000 \%)
$$

Figura 4.17 - Mode-Shape de $\lambda_{10}$ da Matriz [ $\mathrm{J}_{\mathrm{SQv}}$ ] para o Sistema 10 Barras

$$
\text { (jX=10000\%) }
$$

Figura 4.18 - Áreas de Controle de Tensão a partir da Matriz [ $\left.\mathrm{J}_{\mathrm{SQv}}\right]$ para o Sistema

$$
10 \text { Barras com } \mathrm{jX}=10000 \%
$$

Figura 4.19 - Áreas Identificadas pelos Maiores Autovalores da Matriz [VCS] para o Sistema de 39 Barras.

Figura 4.20 - Áreas Identificadas pelos Menores Autovalores da Matriz [ $\mathrm{J}_{\mathrm{SQv}}$ ] para o Sistema de 39 Barras. 144

Figura 4.21 - Fluxogramas Simplificados dos Algoritmos de Busca de Áreas de Controle a partir das Matrizes [VCS] e [ $\left.\mathrm{J}_{\mathrm{SQv}}\right]$ 146

Figura 4.22 - Mode-Shape de $\lambda_{2}$ da Matriz [VCS] para o Sistema IEEE 118 Barras 147

Figura 4.23 - Mode-shape da Figura 4.22 desprezando as barras com módulo inferior a $\sigma=0,4$ 148

Figura 4.24 - Mode-Shape de $\lambda_{2}$ da Matriz [JSQv] para o Sistema IEEE 118 Barras 148

Figura 4.25 - Mode-shape da Figura 4.24 desprezando as barras com módulo inferior a $\sigma=0,5$ 149

Figura 4.26 - Mode-Shape de $\lambda_{2}$ da Matriz [VCS] para o Sistema IEEE 118 Barras ........150

Figura 4.27 - Mode-Shape de $\lambda_{3}$ da Matriz [VCS] para o Sistema IEEE 118 Barras ........150

Figura 4.28 - Mode-Shape de $\lambda_{2}$ da Matriz [J Jov] para o Sistema IEEE 118 Barras..........150

Figura 4.29 - Mode-Shape de $\lambda_{3}$ da Matriz [J Jov] para o Sistema IEEE 118 Barras.........150

Figura 4.30 - Mode-Shape de $\lambda_{1}$ da Matriz [VCS] para o Sistema 18 Barras (jX=0,01\%) . 151

Figura 4.31 - Mode-Shape de $\lambda_{2}$ da Matriz [VCS] para o Sistema 18 Barras $(\mathrm{j} X=0,01 \%)$ 151 
Figura 4.32 - Mode-Shape de $\lambda_{3}$ da Matriz [VCS] para o Sistema 18 Barras

$$
(\mathrm{jX}=0,01 \%)
$$

Figura 4.33 - Mode-Shape de $\lambda_{4}$ da Matriz [VCS] para o Sistema 18 Barras

$$
\text { (jX=0,01\%) }
$$

Figura 4.34 - Áreas de Controle de Tensão a partir da Matriz [VCS] para o Sistema 18 Barras com $\mathrm{jX}=0,01 \%$.

Figura 4.35 - Mode-Shape de $\lambda_{1}$ da Matriz [J $\mathrm{J}_{\mathrm{SQv}}$ ] para o Sistema 18 Barras

$$
(\mathrm{jX}=0,01 \%)
$$

Figura 4.36 - Mode-Shape de $\lambda_{2}$ da Matriz [J $\left.\mathrm{J}_{\mathrm{SQv}}\right]$ para o Sistema 18 Barras

$$
(\mathrm{jX}=0,01 \%)
$$

Figura 4.37 - Mode-Shape de $\lambda_{3}$ da matriz [ $\mathrm{J}_{\mathrm{SQv}}$ ] para o Sistema 18 Barras

$$
(\mathrm{jX}=0,01 \%)
$$

Figura 4.38 - Mode-Shape de $\lambda_{4}$ da Matriz [ $\mathrm{J}_{\mathrm{SQv}}$ ] para o Sistema 18 Barras

$$
(\mathrm{j}=0,01 \%)
$$

Figura 4.39 - Áreas de Controle de Tensão a partir da Matriz [J $\left.\mathrm{J}_{\mathrm{SQv}}\right]$ para o Sistema

$$
18 \text { Barras com jX=0,01\%. }
$$

Figura 4.40 - Mode-Shape de $\lambda_{1}$ da Matriz [VCS] para o Sistema IEEE 118 Barras .........155

Figura 4.41 - Mode-Shape de $\lambda_{2}$ da Matriz [VCS] para o Sistema IEEE 118 Barras ........156

Figura 4.42 - Mode-Shape de $\lambda_{3}$ da Matriz [VCS] para o Sistema IEEE 118 Barras ........156

Figura 4.43 - Mode-Shape de $\lambda_{1}$ da Matriz [J Jov] para o Sistema IEEE 118 Barras.........157

Figura 4.44 - Mode-Shape de $\lambda_{2}$ da Matriz [J J

Figura 4.45 - Mode-Shape de $\lambda_{3}$ da Matriz [J Jov] para o Sistema IEEE 118 Barras.........158

Figura 4.46 - Mode-Shape de $\lambda_{3}$ da Matriz [VCS] para o Sistema IEEE 118 Barras ........159

Figura 4.47 - Áreas de Controle de Tensão a partir da Matriz [VCS] para o Sistema IEEE 118 Barras

Figura 4.48 - Mode-Shape de $\lambda_{3}$ da Matriz [JSQv] para o Sistema IEEE 118 Barras 160

Figura 4.49 - Áreas de Controle de Tensão a partir da Matriz [J $\left.\mathrm{J}_{\mathrm{SQv}}\right]$ para o Sistema IEEE 118 Barras 161

Figura 4.50 - Mode-Shape de $\lambda_{2}$ da Matriz [VCS] para o Sistema 39 Barras 162

Figura 4.51 - Mode-Shape de $\lambda_{3}$ da Matriz [VCS] para o Sistema 39 Barras 162

Figura 4.52 - Mode-Shape de $\lambda_{2}$ da Matriz [J $\mathrm{J}_{\mathrm{sov}}$ ] para o Sistema 39 Barras 163

Figura 4.53 - Mode-Shape de $\lambda_{3}$ da Matriz [J $\mathrm{J}_{\mathrm{SQv}}$ ] para o Sistema 39 Barras 163

Figura 4.54 - Áreas de Controle de Tensão a partir das Matrizes [VCS] e [J Jov] para o Sistema 10 Barras com jX=0,01\% 164

Figura 4.55 - Áreas de Controle de Tensão a partir das Matrizes [VCS] e [Jsov] para o Sistema 10 Barras com jX=10000\% 
Figura 4.56 - Áreas de Controle de Tensão a partir das Matrizes [VCS] e [J Jov] para o Sistema 18 Barras com $\mathrm{jX}=0,01 \%$.

Figura 4.57 - Áreas de Controle de Tensão a partir das Matrizes [VCS] e [Jsav] para o Sistema 18 Barras com $\mathrm{jX}=10000 \%$

Figura 4.58 - Áreas de Controle de Tensão a partir das Matrizes [VCS] e [Jsov] para - Sistema New England 39 Barras

Figura 4.59 - Áreas de Controle de Tensão a partir das Matrizes [VCS] e [Jsov] para o Sistema IEEE 118 Barras. 168

Figura 4.60 - Áreas de Controle de Tensão a partir das Matrizes [VCS] e [Jsav] para o Sistema S/SE Brasileiro 730 Barras 169 


\section{Abreviaturas e Siglas}

\begin{tabular}{|c|c|}
\hline CEPEL & Centro de Pesquisas de Energia Elétrica \\
\hline IEEE & Institute of Electrical and Electronics Engineers \\
\hline RAT ou AVR & Regulador Automático de Tensão ou Automatic Voltage Regulator \\
\hline S/SE & Sul / Sudeste \\
\hline WBE & Weak Break Elimination \\
\hline$P$ & Potência Ativa \\
\hline Q & Potência Reativa \\
\hline S & Potência Aparente \\
\hline$\theta$ & Ângulo da Tensão \\
\hline V & Módulo da Tensão \\
\hline$E$ & Módulo da tensão interna do gerador \\
\hline Xs & Reatância Síncrona \\
\hline$\Delta \mathrm{P}$ & Variação incremental de potência ativa \\
\hline$\Delta Q$ & Variação incremental de potência reativa \\
\hline$\Delta \theta$ & Variação incremental do ângulo da tensão \\
\hline$\Delta \mathrm{V}$ & Variação incremental do módulo da tensão \\
\hline$\Delta \mathrm{E}$ & Variação incremental do módulo da tensão interna do gerador \\
\hline [J] & Matriz Jacobiana \\
\hline$[\mathrm{A}]$ & Submatriz da matriz Jacobiana expandida \\
\hline [B] & Submatriz da matriz Jacobiana expandida \\
\hline$[\mathrm{C}]$ & Submatriz da matriz Jacobiana expandida \\
\hline [D] & Submatriz da matriz Jacobiana expandida \\
\hline [VCS] & Voltage control sensitivity matrix \\
\hline$\partial \mathrm{P} / \partial \theta$ & Derivada parcial da potência ativa em relação ao ângulo da tensão \\
\hline$\partial \mathrm{P} / \partial \mathrm{V}$ & Derivada parcial da potência ativa em relação ao módulo da tensão \\
\hline$\partial \mathrm{P} / \partial \mathrm{t}$ & Derivada parcial da potência ativa em relação ao tape de um LTC \\
\hline$\partial \mathrm{P} / \partial \mathrm{E}$ & $\begin{array}{l}\text { Derivada parcial da potência ativa em relação ao módulo da tensão } \\
\text { interna de um gerador ou compensador síncrono }\end{array}$ \\
\hline$\partial Q / \partial \theta$ & Derivada parcial da potência reativa em relação ao módulo da tensão \\
\hline$\partial \mathrm{Q} / \partial \mathrm{V}$ & Derivada parcial da potência reativa em relação ao módulo da tensão \\
\hline$\partial \mathrm{Q} / \partial \mathrm{t}$ & Derivada parcial da potência reativa em relação ao tape de um LTC \\
\hline$\partial \mathrm{Q} / \partial \mathrm{E}$ & $\begin{array}{l}\text { Derivada parcial da potência reativa em relação ao módulo da tensão } \\
\text { interna de um gerador ou compensador síncrono }\end{array}$ \\
\hline
\end{tabular}




$\begin{array}{ll}\text { nb } & \text { Numero de barras do sistema } \\ \text { nc } & \text { Número de equipamentos controladores de tensão } \\ \text { FP } & \text { Fator de Participação } \\ \lambda & \text { Autovalor } \\ \varphi & \text { Autovetor à direita } \\ \Psi & \text { Autovetor à esquerda }\end{array}$


"A mente que se abre a uma nova idéia jamais voltará ao seu tamanho original"

Albert Einstein (Nobel de Física, 1921) 